\title{
Fluid flow and heat transfer around a confined semi-circular cylinder: Onset of vortex shedding and effects of Reynolds and Prandtl numbers
}

\author{
Anuj Kumar ${ }^{a}$, Amit Dhiman ${ }^{a}$, László Baranyi ${ }^{\mathrm{b}, *}$ \\ a Department of Chemical Engineering, Indian Institute of Technology Roorkee, Roorkee 247 667, India \\ ${ }^{\mathrm{b}}$ Department of Fluid and Heat Engineering, Institute of Energy Engineering and Chemical Machinery, University of Miskolc, 3515 Miskolc-Egyetemváros, Hungary
}

\section{A R T I C L E I N F O}

\section{Article history:}

Received 29 March 2016

Received in revised form 7 June 2016

Accepted 10 June 2016

Available online $\mathrm{xxxx}$

\section{Keywords:}

Confined flow

Critical Reynolds number

Prandtl number

Semi-circular cylinder

Strouhal number

Unsteady flow

\begin{abstract}
A B S T R A C T
Flow and heat transfer characteristics around a semi-circular cylinder placed in a confined channel are investigated in the unsteady regime. The two-dimensional simulations are carried out for varying values of control parameters: Reynolds number $(\mathrm{Re})=50-200$ and Prandtl number $(\mathrm{Pr})=0.7,10$ and 100 at a fixed blockage ratio of $25 \%$ for Newtonian constant-property fluid. Continuity, Navier-Stokes and energy equations with appropriate boundary conditions are solved using the commercial computational fluid dynamics solver Ansys Fluent. The transition from steady to time-periodic flow occurs between $\operatorname{Re}=69$ and 70. The effect of Prandtl number on Nusselt number is pronounced; the ratio of Nusselt number values belonging to $\operatorname{Pr}=100$ and those belonging to $\operatorname{Pr}=0.7$ ranges from 6.3 to 6.5 over the Reynolds number domain investigated. Finally, the present numerical results are used to develop drag coefficient, Strouhal number and Nusselt number correlations.
\end{abstract}

(c) 2016 Elsevier Ltd. All rights reserved.

\section{Introduction}

Analogous to a circular cylinder, confined flow and heat transfer around a semi-circular cylinder have variety of engineering applications such as cooling of electronic components and chips of various shapes, pin type heat exchange systems, thermal processing of foodstuffs, vortex flow meters, and others [1-5]. Besides, a semicircular cylinder offers space economy in terms of the specific heat transfer area. In spite of such widespread applications, limited information is available in the open literature on the confined flow around and heat transfer from a semi-circular cylinder. We have recently systematically presented and discussed various studies on the flow and heat transfer characteristics in a channel with a built-in semi-circular cylinder [6-8]; Kumar and Dhiman [6] and Kumar et al. [7] investigated the confined forced flow and heat transfer around a semi-circular cylinder, albeit at low Reynolds numbers (Re up to 40). This motivated us to examine the confined forced convection heat transfer from a semi-circular cylinder in the unsteady regime (or at intermediate Re). On the other hand, extensive numerical/experimental literature is available on the forced flow and heat transfer around a semi-circular cylinder in the unconfined domain [9-24]. Among these studies, Kiya and Arie

\footnotetext{
* Corresponding author.

E-mail addresses: dhimuamit@rediffmail.com, amitdfch@iitr.ac.in (A. Dhiman), arambl@uni-miskolc.hu (L. Baranyi).
}

[9], Boisaubert et al. [10], Coutanceau et al. [11], Sophy et al. [12], Koide et al. [13], Koide et al. [14], Chandra and Chhabra $[15,16]$, Gode et al. [17], Bhinder et al. [18] and Chatterjee et al. [19] investigated the unsteady flow around a semi-circular cylinder. Forbes and Schwartz [20] and Chandra and Chhabra [21,22] determined the effects of control parameters on a semi-circular obstacle in the steady regime (or at low Re). Tiwari and Chhabra [23] investigated the influence of flow and heat transfer parameters on flow around and heat transfer from a semi-circular cylinder for power-law fluids in the steady regime $(\operatorname{Re}=0.01-30$ and $\operatorname{Pr}=1-$ 100). The classical inverse variation in the value of the drag coefficient with Re is reported. In a recent study, Chatterjee and Mondal [24] studied the mixed convection heat transfer across a semicircular cylinder in the unsteady regime for $R e=50-150$ at a fixed Prandtl number $(\mathrm{Pr}=0.71)$. Considerable differences in the global flow and heat transfer quantities are observed for the range of settings investigated.

Thus, as far as we know, no one has investigated the unsteady momentum and heat transfer around a confined semi-circular cylinder in a channel, in spite of its many engineering applications [1-5]. In the confined configuration, forced convection heat transfer phenomena are noticeably influenced by the wall confinement or blockage ratio (defined as the ratio of a semi-circular cylinder's diameter $(D)$ to the channel transverse height $(H)$, that is $\beta=D / H)$ in addition to the values of Re and Pr. The present work aims to fill these gaps in the confined flow configuration for the forced flow 


\begin{tabular}{|c|c|c|c|}
\hline \multicolumn{4}{|c|}{ Nomenclature } \\
\hline C & heat capacity of fluid $\left(\mathrm{J} \mathrm{kg}^{-1} \mathrm{~K}^{-1}\right)$ & $T$ & absolute temperature $(\mathrm{K})$ \\
\hline$C_{D}$ & total drag coefficient $\left(=F_{D} /\left(1 / 2 \rho U_{\infty}^{2} D\right)\right)$ & $T_{p}$ & dimensionless time period of one periodic cycle \\
\hline$C_{L}$ & total lift coefficient $\left(=F_{L} /\left(1 / 2 \rho U_{\infty}^{2} D\right)\right)$ & $T_{\infty}$ & fluid temperature at the inlet $(\mathrm{K})$ \\
\hline CV & control volume & $T_{w}$ & surface temperature of the semi-circular obstacle $(\mathrm{K})$ \\
\hline$D$ & diameter of the semi-circular cylinder (m) & $\mathbf{U}$ & dimensionless velocity vector $\left(=\mathrm{U}^{*} / U_{\infty}\right)$ \\
\hline$F$ & vortex shedding frequency $\left(\mathrm{s}^{-1}\right)$ & $U_{\infty}$ & average velocity at the inlet $\left(\mathrm{m} \mathrm{s}^{-1}\right)$ \\
\hline$F_{D}$ & $\begin{array}{l}\text { drag force per unit length of the semi-circular obstacle } \\
\left(\mathrm{N} \mathrm{m}^{-1}\right)\end{array}$ & $\begin{array}{l}U_{x}, U_{y} \\
x, y\end{array}$ & $\begin{array}{l}x \text { - and } y \text {-components of dimensionless velocities } \\
\text { dimensionless streamwise and transverse coordinates }\end{array}$ \\
\hline$F_{L}$ & $\begin{array}{l}\text { lift force per unit length of the semi-circular obstacle } \\
\left(\mathrm{N} \mathrm{m}^{-1}\right)\end{array}$ & $X_{d}$ & $\begin{array}{l}\left(=x^{*} / D, y^{*} / D\right) \\
\text { downstream distance }(\mathrm{m})\end{array}$ \\
\hline $\begin{array}{l}h \\
\bar{h}\end{array}$ & $\begin{array}{l}\text { local heat transfer coefficient }\left(\mathrm{W} \mathrm{m} \mathrm{m}^{-2} \mathrm{~K}^{-1}\right) \\
\text { average heat transfer coefficient }\left(\mathrm{W} \mathrm{m} \mathrm{m}^{-2} \mathrm{~K}^{-1}\right)\end{array}$ & $X_{u}$ & upstream distance $(\mathrm{m})$ \\
\hline$H$ & height of the computational domain (m) & \multicolumn{2}{|c|}{ Greek symbols } \\
\hline$k$ & thermal conductivity of fluid ( $\mathrm{W} \mathrm{m}^{-1} \mathrm{~K}^{-1}$ ) & $\beta$ & blockage ratio $(=D / H)$ \\
\hline$L$ & length of the domain $(\mathrm{m})$ & $\delta$ & size of the CV clustered around a semi-circular \\
\hline $\mathrm{Nu}_{\mathrm{L}}$ & local Nusselt number $(=h D / k)$ & & cylinder $(\mathrm{m})$ \\
\hline $\mathrm{Nu}$ & average Nusselt number $(=\bar{h} D / k)$ & $\theta$ & dimensionless temperature $\left(=\left(T-T_{\infty}\right) /\left(T_{w}-T_{\infty}\right)\right)$ \\
\hline$p$ & dimensionless pressure $\left(=p^{*} /\left(\rho U_{\infty}^{2}\right)\right)$ & $\mu$ & dynamic viscosity of fluid ( $\mathrm{Pa} \mathrm{s})$ \\
\hline $\operatorname{Pr}$ & Prandtl number $(=\mu C / k)$ & $\rho$ & fluid density $\left(\mathrm{kg} \mathrm{m}^{-3}\right)$ \\
\hline $\operatorname{Re}$ & Reynolds number $\left(=D U_{\infty} \rho / \mu\right)$ & & \\
\hline St & Strouhal number $\left(=f D / U_{\infty}\right)$ & \multirow{3}{*}{\multicolumn{2}{|c|}{$\begin{array}{l}\text { Superscript } \\
* \quad \text { dimensional value }\end{array}$}} \\
\hline$t$ & dimensionless time $\left(=t^{*} /\left(D / U_{\infty}\right)\right)$ & & \\
\hline$\Delta t$ & dimensionless time-step & & \\
\hline
\end{tabular}

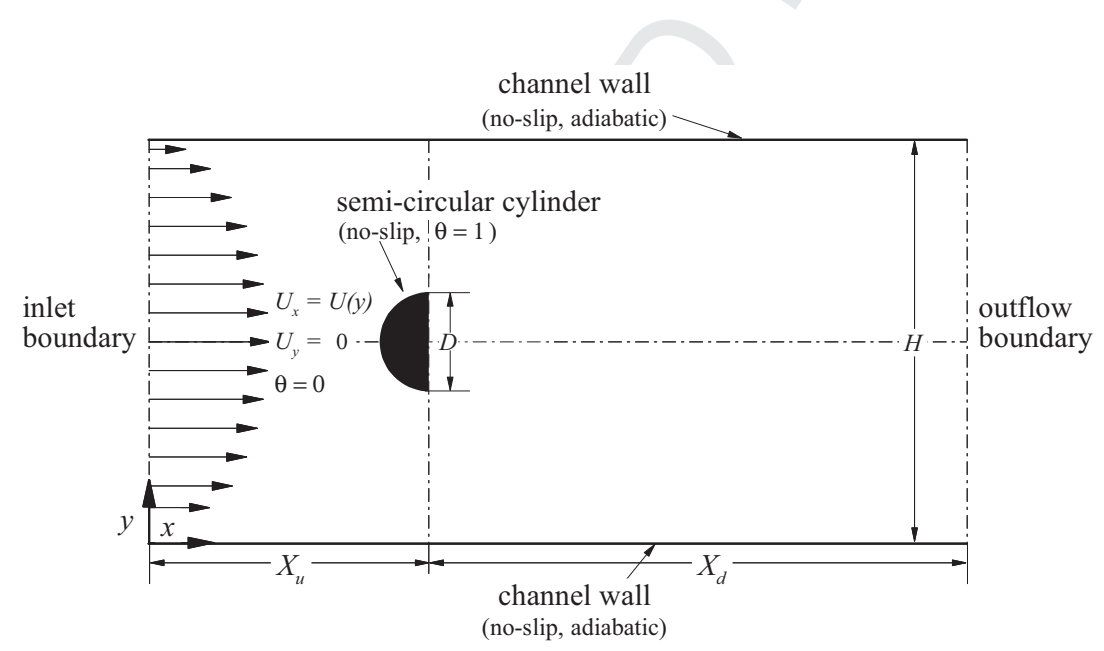

Fig. 1. Schematic diagram.

around and heat transfer from a semi-circular cylinder in the unsteady regime. The effect of various values of control parameters ( $R e$ and Pr) on the engineering output parameters (such as drag coefficient, Nusselt and Strouhal numbers) and temporal variation in the values of drag and lift coefficients and Nusselt number are discussed. Instantaneous flow and thermal patterns around a semi-circular cylinder are also presented. Lastly, simple expressions of drag coefficient, Strouhal number and Nusselt number are determined.

\section{Problem formulation}

Confined laminar flow of constant property incompressible Newtonian fluids in a channel with a built-in heated semicircular cylinder is shown schematically in Fig. 1. The long semicircular cylinder is exposed to a fully developed velocity field with average velocity $U_{\infty}$ and uniform temperature $T_{\infty}$ at the inlet. The semi-circular cylinder is located symmetrically on the centerline of the channel at an upstream distance of $X_{u}$ from the inlet and at a
Table 1

Choice of upstream and downstream distances for $\operatorname{Re}=200$ and $\operatorname{Pr}=100$.

\begin{tabular}{llllll}
\hline$X_{u} / D$ & $C_{D}$ & $\mathrm{Nu}$ & $X_{d} / D$ & $C_{D}$ & $\mathrm{Nu}$ \\
\hline 30 & 2.9501 & 51.5860 & 100 & 2.9565 & 51.6080 \\
45 & 2.9765 & 51.1819 & 120 & 2.9765 & 51.1819 \\
60 & 2.9958 & 50.7871 & 140 & 3.0000 & 50.6999
\end{tabular}

Table 2

Choice of grid size and time step for $\operatorname{Re}=200$ and $\operatorname{Pr}=100$.

\begin{tabular}{llllll}
\hline Grid & $C_{D}$ & $\mathrm{Nu}$ & $\Delta t$ & $C_{D}$ & $\mathrm{Nu}$ \\
\hline G1 & 2.9516 & 51.5871 & 0.1 & 2.9690 & 51.1020 \\
G2 & 2.9765 & 51.1819 & 0.05 & 2.9980 & 51.2535 \\
G3 & 2.9999 & 50.6074 & 0.01 & 2.9765 & 51.1819
\end{tabular}



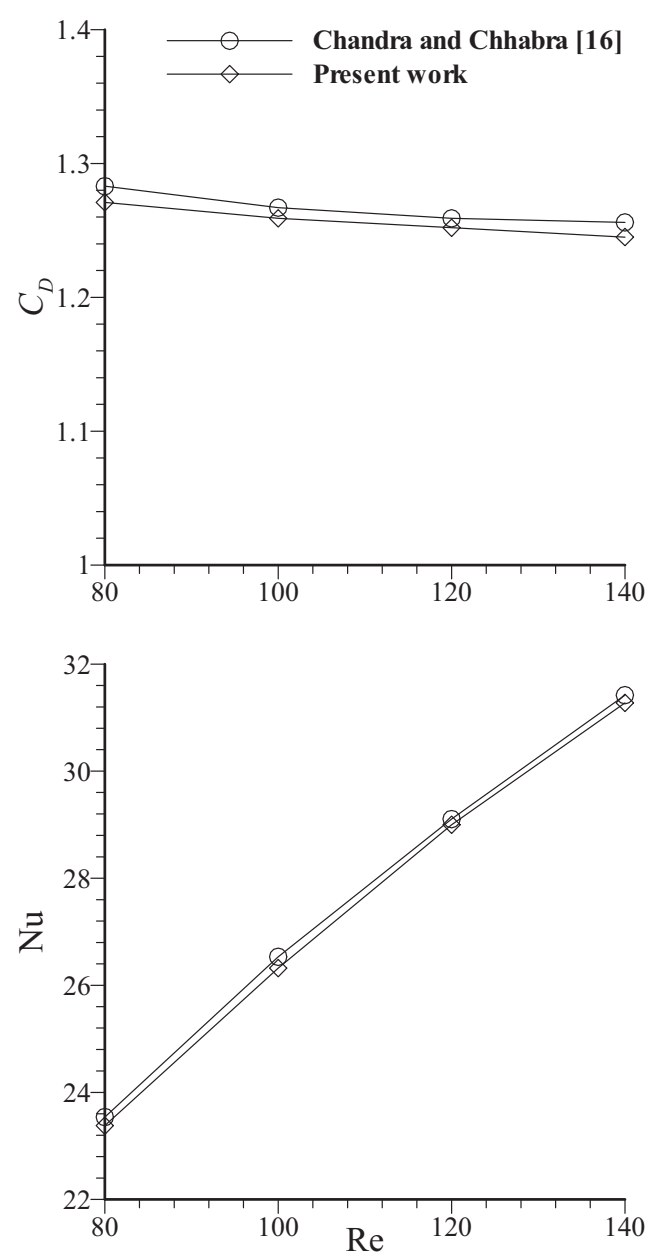

Fig. 2. $C_{D}$ and $\mathrm{Nu}$ for a semi-circular cylinder in the unsteady unconfined flow regime at $\operatorname{Pr}=50$; present results and Chandra and Chhabra [16]. downstream distance of $X_{d}$ from the outlet measured from the flat side of the cylinder. The total length of the computational domain is $L\left(=X_{u}+X_{d}\right)$ in the axial direction and the height of the computational domain is $H$ in the lateral direction. The blockage ratio $\beta=D /$ $H$, where $D$ is the diameter of the semi-circular cylinder, is fixed at 0.25 .

The dimensionless forms of the continuity, Navier-Stokes and energy equations are represented by Eqs. (1)-(3), respectively.

$\nabla \cdot \mathrm{U}$

$\frac{D U}{D t}=-\nabla p+\frac{1}{\operatorname{Re}} \nabla^{2} \mathrm{U}$

$\frac{D \theta}{D t}=\frac{1}{\operatorname{RePr}} \nabla^{2} \theta$

In Eq. (2) the free convection term is neglected because it is much smaller for our case than the forced convection. The importance of free convection relative to forced convection is characterized by the buoyancy parameter, the Richardson number $\mathrm{Ri}=\mathrm{Gr} / \operatorname{Re}^{2}=g \beta_{v} \Delta T D / U_{\infty}^{2}$, where Gr, $g, \beta_{\nu}, \Delta T, D$ and $U_{\infty}$ are the Grashoff number, acceleration due to gravity, coefficient of volumetric expansion, temperature difference, diameter of the semicircular cylinder and fluid velocity at the inlet, respectively. Ri is calculated using the experimental value of $\beta_{v}$ [25] and the Ri ranges here from 0.014 (for engine oil, $\operatorname{Pr}=100$ ) to 0.066 (for air, $\operatorname{Pr}=0.7$ ). Due to such low values obtained for Ri, the effect of the cross-buoyancy term is neglected in Eq. (2). In Eq. (3) the viscous dissipation is neglected, as is typical for lower Reynolds number investigations. Since the Prandtl number Pr occurs only in the energy Eq. (3), Pr affects the temperature distribution and heat transfer, but not the flow properties.

The following dimensionless boundary conditions are given for the current flow situation: at the channel inlet, a fully developed velocity profile is utilized [6-8],

$U_{x}=1.5\left[1-(|1-2 \beta y|)^{2}\right](0 \leqslant y \leqslant H / D), \quad U_{y}=0$ and $\theta=0$.
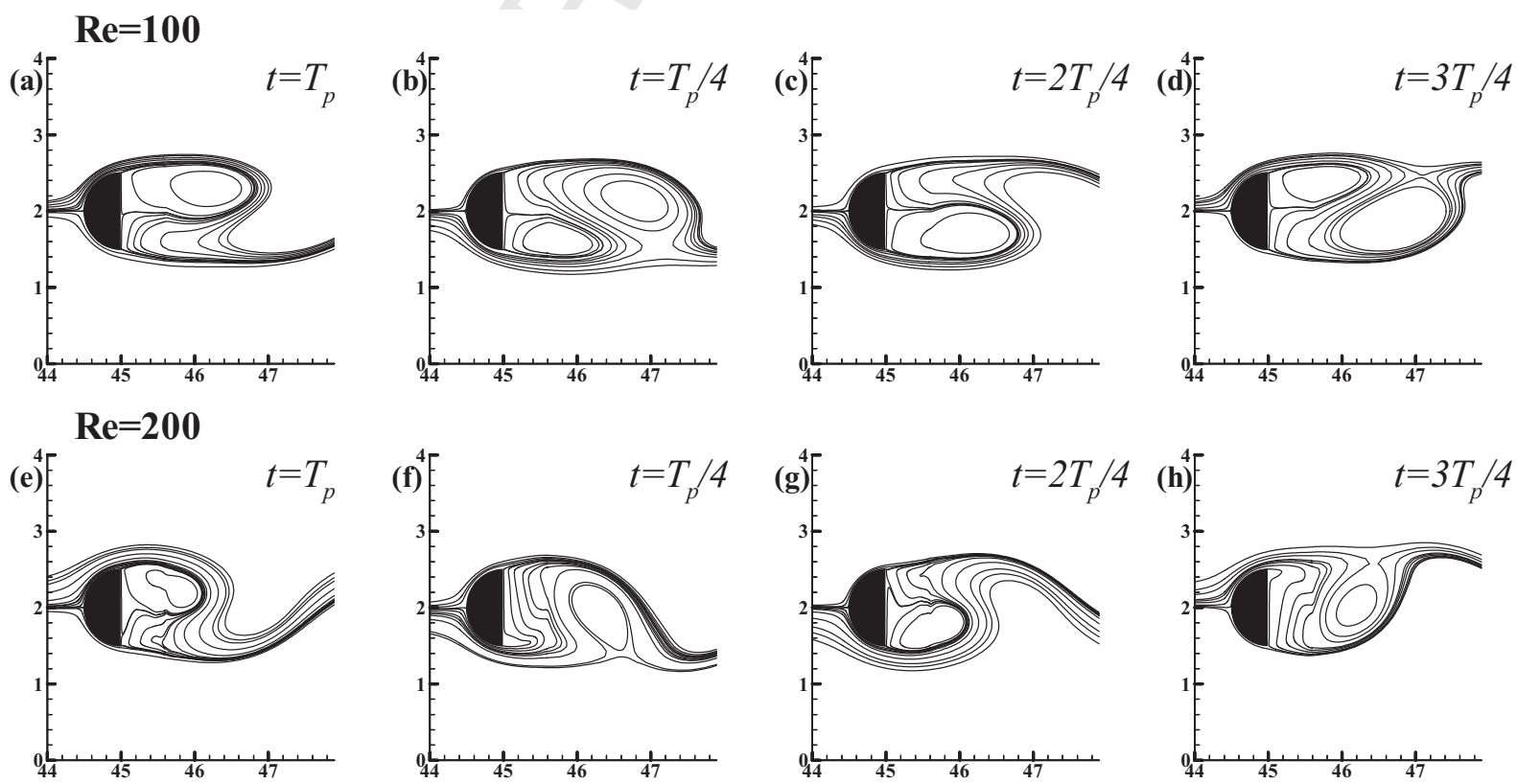

Fig. 3. Instantaneous streamlines at different phases of vortex shedding for $(a-d) R e=100$ and $(e-h) R e=200$ in the time-periodic regime. 

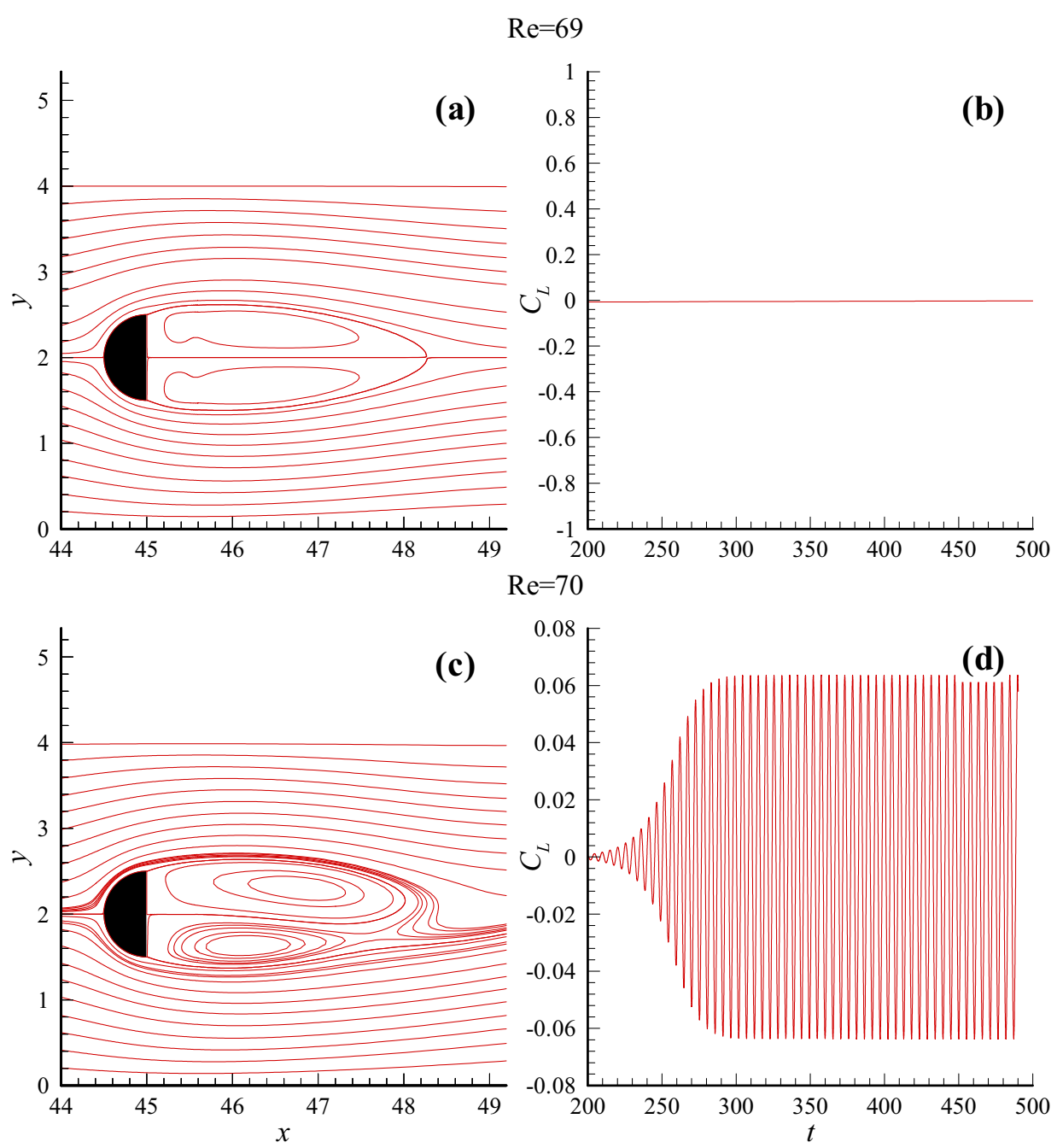

Fig. 4. Onset of transition from a steady to a time-periodic regime: (a, c) streamlines and (b, d) time histories of lift coefficients at the Re of 69 and 70.

On the top and bottom walls of the domain, $U_{x}=0, U_{y}=0$ (noslip) and $\partial \theta / \partial y=0$ (adiabatic). On the surface of a semi-circular cylinder, $U_{x}=0, U_{y}=0$ (no-slip) and $\theta=1$ (uniform wall temperature). At the channel exit, $\partial U_{x} / \partial x=0, \partial U_{y} / \partial x=0$ and $\partial \theta / \partial x=0$. Pressure boundary conditions are not explicitly required, because the solver extrapolates the pressure from the interior [26].

\section{Description of numerical solution procedure and the grid, domain and time step dependence studies}

A non-uniform computational grid was created (see [7]) using the commercial grid generator Gambit. The grid is fine near the semi-circular cylinder and near the top and bottom channel walls, whereas a coarse grid is used far away from the cylinder and the domain walls. For instance, a fine grid with a cell size $(\delta)$ of $0.01 D$ (the number of control volumes (CVs) on a semi-circular cylinder is 340) is clustered around a semi-circular cylinder and near the walls, and the largest grid size used is $0.5 D$.

The governing equations (Eqs. (1)-(3)) are solved with the specified boundary conditions using the commercial computational fluid dynamics solver Ansys Fluent [26]. A second order upwind scheme is used to discretize convective terms of momentum and energy equations, while the diffusive terms are discretized by a central difference scheme. The SIMPLE method was used for the pressure-velocity decoupling. The ensuing algebraic equations are solved by the Gauss-Siedel iterative scheme in combination with the Algebraic Multi-Grid scheme until the absolute convergence criterion of $10^{-15}$ is satisfied for continuity, velocities and energy. Also, the solution is assumed to have converged when it shows at least 10 constant amplitude cycles in the time history profiles of output parameters [27-32]. The geometrical parameters such as upstream and downstream distances, grid size and time step were selected after a thorough study and these details are provided underneath.

The effect of upstream distance $\left(X_{u} / D\right)$ on the values of drag coefficient $\left(C_{D}\right)$ and average Nusselt number $(\mathrm{Nu})$ is explored for $X_{u} / D=30,45$ and 60 at the highest value of $\operatorname{Re}(200)$ and $\operatorname{Pr}(100)$ studied (Table 1 ). The relative differences in the values of $C_{D}$ and $\mathrm{Nu}$ for $X_{u} / D=30$ were found to be less than $1.6 \%$ for both $C_{D}$ and $\mathrm{Nu}$, with respect to the values at $X_{u} / D=60$, whereas for $X_{u} / D=45$ the corresponding differences were found to be less than $1 \%$ for both $C_{D}$ and Nu. Hence, the dimensionless upstream distance of 45 seems to be adequate to avoid entrance effects. Similarly, computations were carried out to determine the effect of downstream distance $\left(X_{d} / D\right)$ on the physical output parameters by considering $X_{d} / D=100,120$ and 140 for $\operatorname{Re}=200$ and $\operatorname{Pr}=100$ (Table 1 ). The relative deviations in the values of $C_{D}$ for $X_{d} / D=100$ were found to be less than $1.5 \%$ with respect to the values at $X_{d} / D=140$, while for $X_{d} / D=120$ the corresponding differences were observed to be 

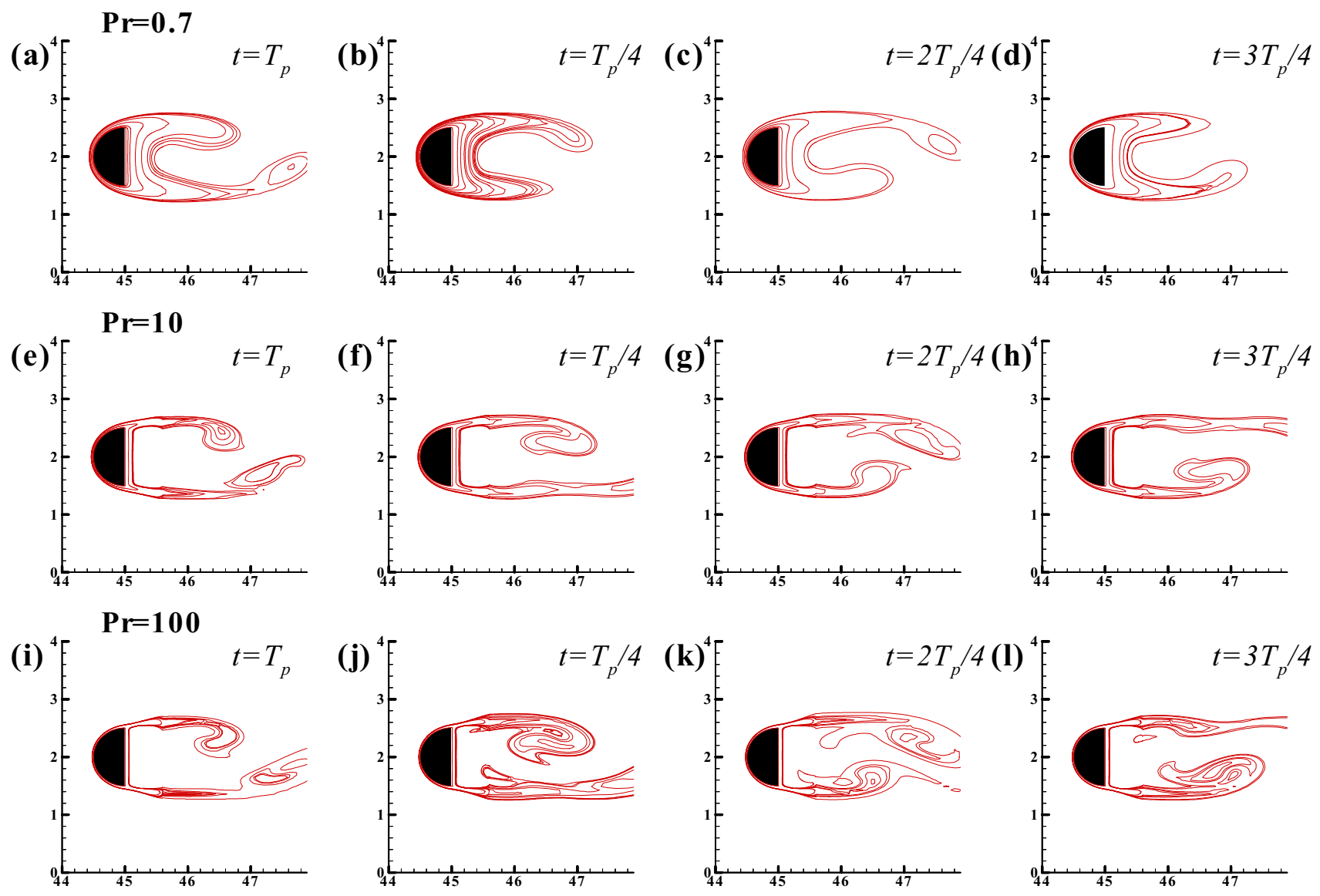

Fig. 5. Instantaneous isotherm contours at different phases of vortex shedding for $\mathrm{Re}=100$ at different Prandtl numbers.

about $0.8 \%$. The relative changes in the values of $\mathrm{Nu}$ for $X_{d} / D=100$ and 120 were found to be about 1.8 and less than $1 \%$, respectively, with respect to the values at $X_{d} / D=140$. Hence, the dimensionless downstream distance of 120 seems to be adequate to avoid end effects.For a grid resolution study (Table 2), a careful examination of physical parameters was undertaken by considering three grids consisting of 43,436 (G1), 103,579 (G2) and 127,691 (G3) cells (with $250(\delta / D=0.1), 340(\delta / D=0.01)$ and $400(\delta / D=0.008) \mathrm{CVs}$ allocated on the surface of a semi-circular cylinder, respectively), at $\operatorname{Re}=200$ and $\operatorname{Pr}=100$. The relative percentage differences in the values of $C_{D}$ and $\mathrm{Nu}$ for the grid $\mathrm{G} 2$ were found to be only about 0.8 and less than $1.1 \%$, respectively, as compared to the values for the grid G3. Thus, the optimized grid G2 is used for further calculations of output flow and heat transfer parameters.

Finally, the optimum value of non-dimensional time step $(\Delta t)$ was fixed for the present study (Table 2). For this, numerical results were obtained for the three non-dimensional time steps of 0.1 $\left(\Delta t_{1}\right), 0.05\left(\Delta t_{2}\right)$ and $0.01\left(\Delta t_{3}\right)$ for the optimized grid G2 (103,579 cells), dimensionless upstream distance of 45 and downstream distance of 120 at $\operatorname{Re}=200$ and $\operatorname{Pr}=100$. The relative differences in the values of $C_{D}$ and $\mathrm{Nu}$ for the $\Delta t_{2}$ were found to be about $0.7 \%$ and $0.2 \%$, respectively, as compared to the values at $\Delta t_{3}$. Thus, the non-dimensional time-step of $0.01\left(\Delta t_{3}\right)$ is used for further calculations.

\section{Results and discussion}

The computations were carried out for the parameter domain of 16 Re values ( 50 to 200 in intervals of 10 ), and $3 \operatorname{Pr}$ values (0.7, 10 and 100, for air, water and engine oil as working fluids, respectively) at a blockage ratio of $25 \%$ [7]. The fixed blockage ratio of $25 \%$ is considered because extensive studies [32-38] on various other shapes of bodies are available for this ratio. It is also to be noted that for an unconfined stationary circular cylinder 3D instability occurs at around $\operatorname{Re}=188.5$ (mode A instability) for Newtonian constant property fluid [39]. Despite this fact, carrying out the present 2D simulations up to $\operatorname{Re}=200$ is justified because fluid flow stability is stronger for a confined domain [6] than an unconfined domain [21].

Fig. 2 shows a comparison of the present unconfined values of $C_{D}$ and $\mathrm{Nu}$ with the reliable unconfined values of Chandra and Chhabra [16] for a semi-circular cylinder in the unsteady regime $(\operatorname{Re}=80-140)$ at $\operatorname{Pr}=50$. A maximum deviation of less than $1 \%$ is noted between the present values of $C_{D}$ and $\mathrm{Nu}$ and those in [16]. In addition, the current numerical methodology was benchmarked with Chandra and Chhabra $[21,40]$ in our recent studies $[6,7]$ on the confined flow around a semi-circular cylinder in the steady regime; it is not repeated here for brevity. Furthermore, this methodology is benchmarked for the flow around a cylinder of square cross-section inclined at an angle of incidence of $45^{\circ}$ in the channel with $25 \%$ blockage [41]. Thus, the present numerical solution procedure can be considered trustworthy.

\subsection{Flow patterns}

The flow was found to be steady, as two symmetric vortices are formed behind a semi-circular cylinder, for the range of Re up to 69 . This is a steady state due to the reduction of disturbances and the increase in flow stability due to the confined walls (in unconfined flow, a value of 39.5 has been identified [21]). Not surprisingly, the size of these symmetric vortices increases linearly with increasing Re. On further increasing the value of $\operatorname{Re}(\geqslant 70)$, 

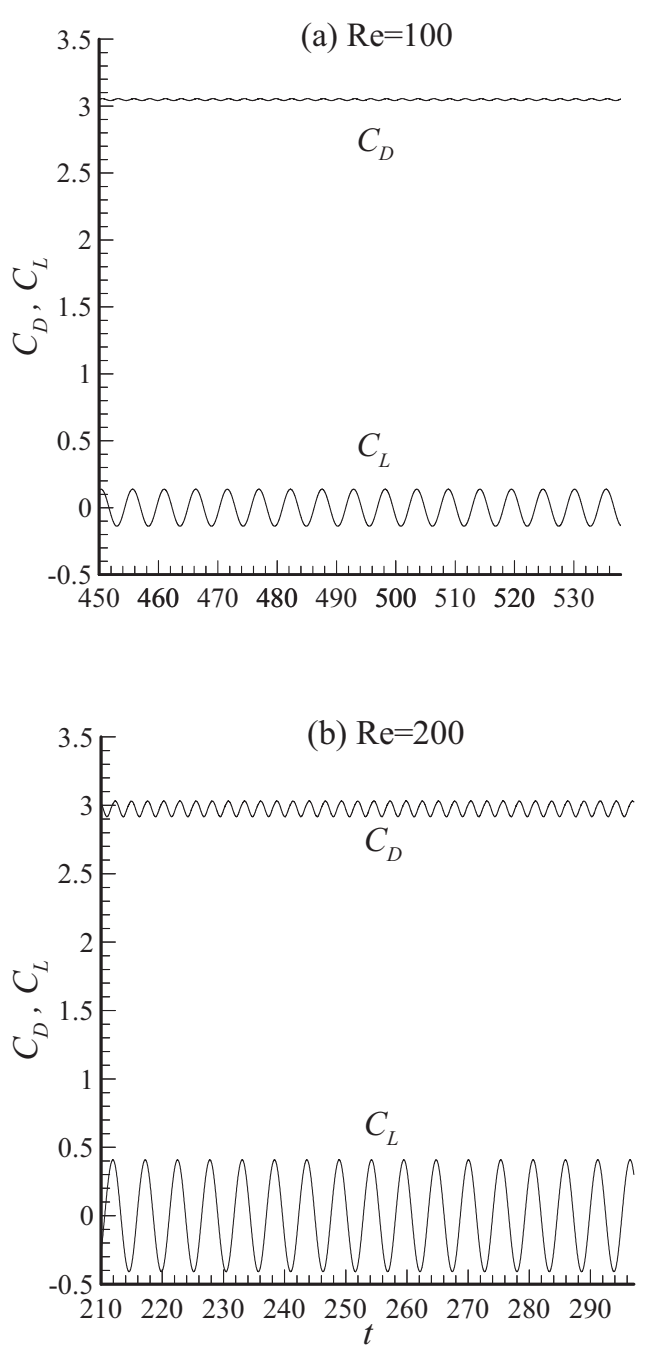

Fig. 6. Time history of drag and lift coefficients for (a) $R e=100$ and (b) 200 .

the flow field shows non-symmetrical behavior. For instance, Fig. 3a-h represent the instantaneous streamlines around a confined semi-circular cylinder for the flow of Newtonian fluids at $\operatorname{Re}=100$ and 200. These figures show the periodic variations in the vortices behind the semi-circular cylinder at the four time instances of a complete periodic cycle (in the figure $T_{p}$ is the time period for vortex shedding). The appearance of an instantaneous or time dependent small wake behind the semi-circular cylinder that can be seen in Fig. $3 a-h$ is similar to the wake in the unsteady unconfined flow regime [16,27-32].

Furthermore, the onset of transition from a steady to a timeperiodic regime (or the onset of vortex shedding) is determined by means of streamlines (Fig. 4a and c) and time history of the lift coefficient (Fig. 4b and d), at $\operatorname{Pr}=0.7$. It is found that at $\operatorname{Re}=69$ the value of the lift coefficient remains at zero throughout the very long time period investigated, as expected for steady symmetric flow. For $\operatorname{Re}=70$, however, the flow becomes unsteady and after some transition, periodic flow develops due to vortex shedding. These findings suggest that the critical Reynolds number lies between 69 and 70 for the flow situation studied.

\subsection{Isotherm patterns}

Fig. 5 shows the isotherm contours around a semi-circular cylinder at different phases of vortex shedding for $\operatorname{Pr}=0.7,10$

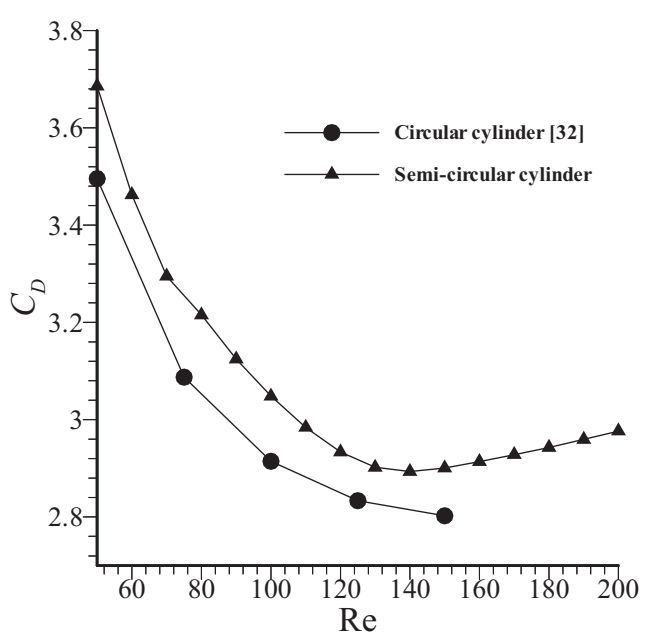

Fig. 7. Variation in time-mean value of the drag coefficient with Re for a semicircular cylinder with values of Bijjam and Dhiman [32] for a circular cylinder in a confined domain of $25 \%$ blockage.

and 100 at $R e=100$. It is observed that the curved surface (upstream surface) of the semi-circular cylinder has the maximum crowding of the temperature contours (due to the thin boundary layer) as compared to the flat (or downstream) surface at constant Re and Pr. An increase in the Pr increases the crowding of isotherms around both the flat and curved parts of the semi-circular cylinder. The isotherms are confined to a smaller region at higher values of $\mathrm{Pr}$, thereby suggesting the bulk of the resistance to heat transfer is confined to a thin layer of fluid. This is clearly due to the thinning of the thermal boundary layer with increasing $\mathrm{Pr}$ [27-32]. The temperature contours behind the confined semicircular cylinder form a similar pattern to the Kármán vortex street.

273

274

275

276

277

278

279

280

281

282

283

284

285

\subsection{Drag and lift coefficients}

The temporal variations in the values of drag and lift coefficients in the periodic regime $(\operatorname{Re} \geqslant 70)$ at the $\operatorname{Re}$ of 100 and 200 are shown in Fig. $6 a$ and b, respectively. It can be seen in Fig. $6 a$ and $b$ that the amplitude of both the lift and drag coefficient increases slightly with increasing Re.

Fig. 7 presents the time-mean values of drag coefficient against Re for steady ( $\operatorname{Re} \leqslant 69)$ as well as time-periodic $(\operatorname{Re}=70-200)$ flows. In the steady regime (that is, up to $R e=69$ ), as expected, the total drag coefficient decreases with Re. In the time-periodic regime, the time-mean value of the drag coefficient is calculated by averaging at least 10 cycles beyond the time at which the asymptotic shedding frequency of Kármán vortex is achieved. It was found that the time-mean value of drag coefficient decreases sharply with increasing Re for a semi-circular cylinder for Re $\leqslant$ 140. To show the similarity with the confined flow around a circular cylinder, Fig. 7 also includes the values of the drag coefficient for a circular cylinder in a channel with blockage of 25\% [32]. The drag coefficient is found to be higher for a semi-circular cylinder than for a circular cylinder over the range of settings investigated. This phenomenon is similar to the unconfined flow over semi-circular, square and circular cylinders reported elsewhere [16,27-32]. In contrast, for the Re range 150-200; the time-mean value of the drag coefficient increases moderately with increasing Re.

Furthermore, after a thorough investigation, the following simple expression (Eq. (4)) was developed to represent the total 


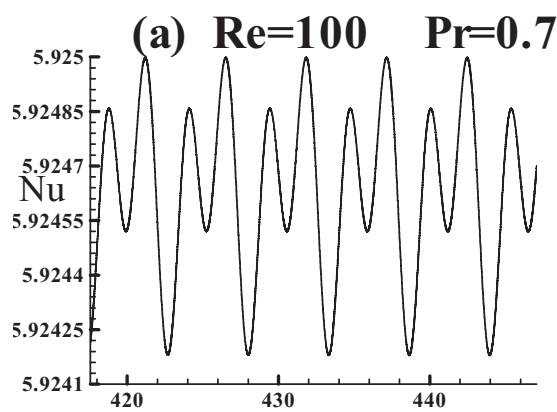

(d) $\operatorname{Re}=150 \quad \operatorname{Pr}=0.7$
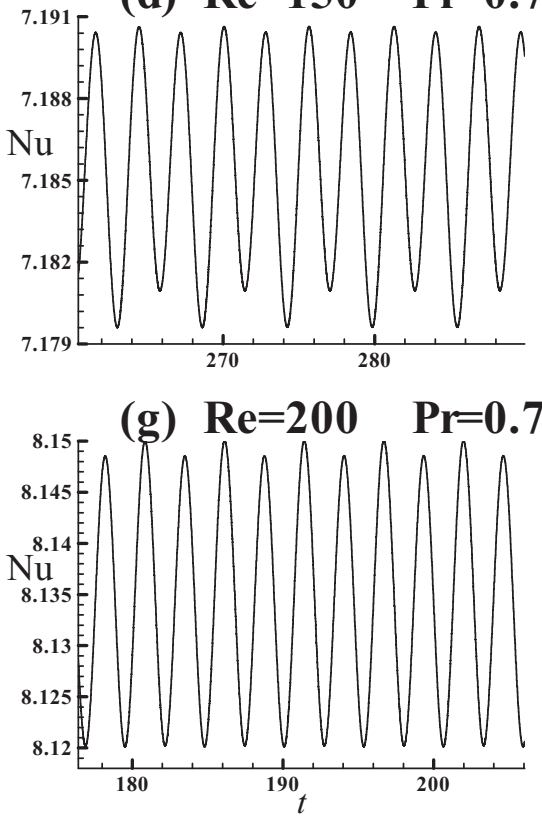

(b) $\operatorname{Re}=100 \quad \operatorname{Pr}=10$

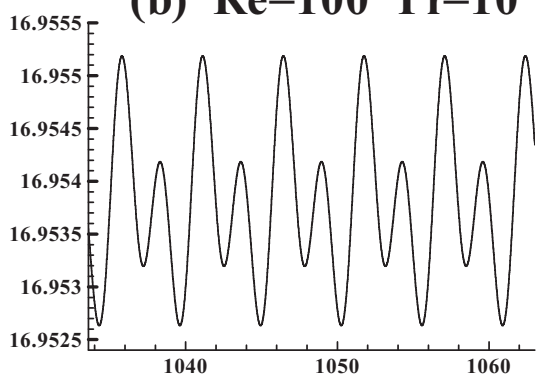

(e) $\operatorname{Re}=150 \quad \operatorname{Pr}=10$

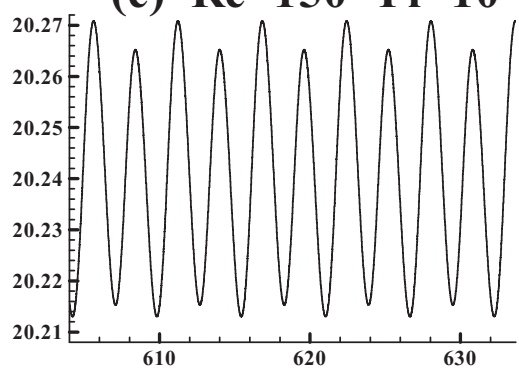

(h) $\operatorname{Re}=200 \quad \operatorname{Pr}=10$

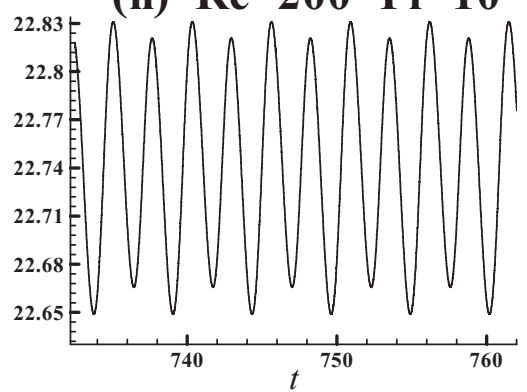

(c) $\operatorname{Re}=100 \quad \operatorname{Pr}=100$

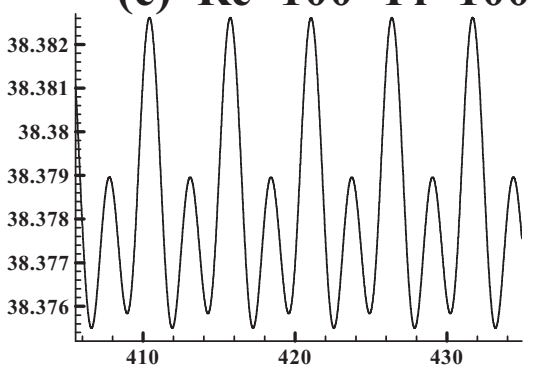

(f) $\operatorname{Re}=150 \quad \operatorname{Pr}=100$

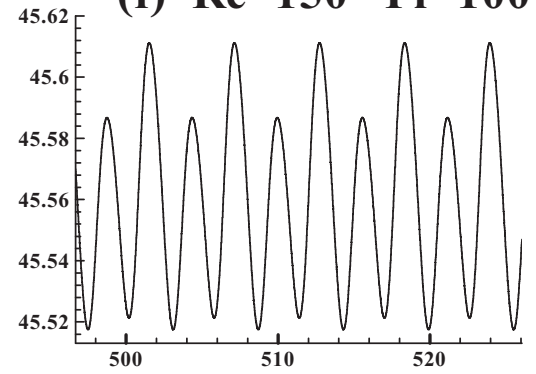

(i) $\operatorname{Re}=200 \quad \operatorname{Pr}=100$

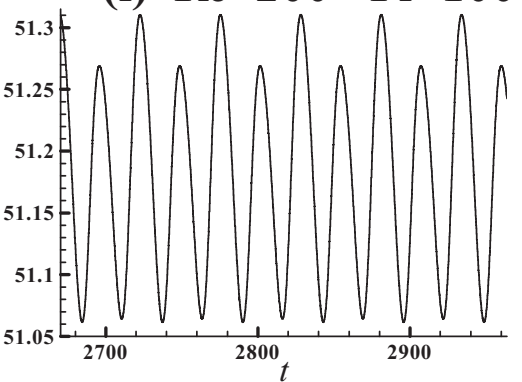

Fig. 8. Time history of Nusselt number for $\operatorname{Pr}=0.7$ (a, d, g), $\operatorname{Pr}=10$ (b, e, h) and $\operatorname{Pr}=100$ (c, f, i) at $\operatorname{Re}=100,150,200$.

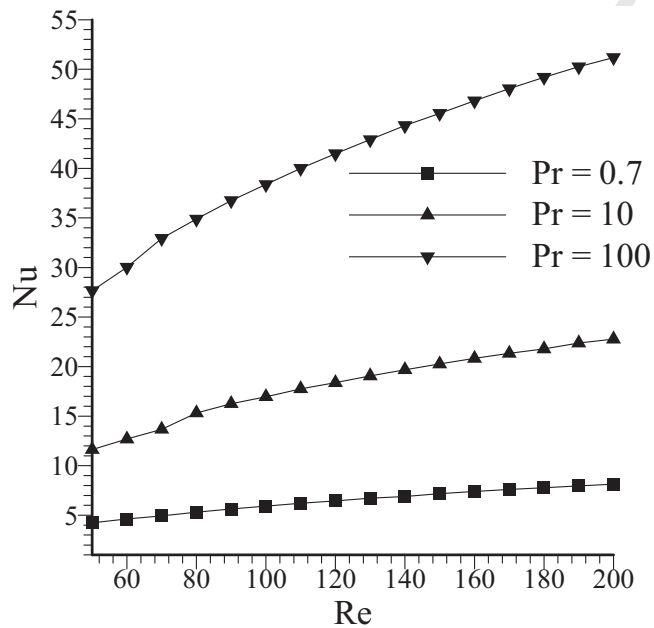

Fig. 9. Variation in the average cylinder Nusselt number against $\operatorname{Re}$ at $\operatorname{Pr}=0.7,10$ and 100 .

drag coefficient as a function of Re (for the Re range 50-200) for a blockage ratio of $25 \%$.

$C_{D}=7.1918+0.0286 \mathrm{Re}-0.7016 \mathrm{Re}^{0.5}$

This correlation has a maximum deviation of less than $0.9 \%$ at $\operatorname{Re}=70$; however, the average deviation is below $0.5 \%$ for $50<\operatorname{Re} \leqslant 200$ with the present computed drag coefficient values.

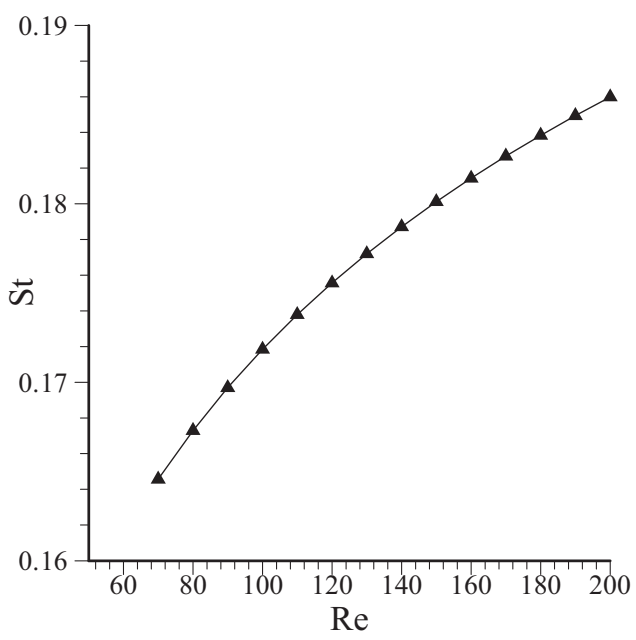

Fig. 10. Variation in Strouhal number with Re for a semi-circular cylinder in the confined domain of $25 \%$ blockage.

\subsection{Nusselt number}

Similarly to drag and lift coefficients shown in Section 4.3, the temporal variation in the values of Nusselt numbers for the Re of 100,150 and 200 at the Pr of $0.7,10$ and 100 is given in 
Fig. 8a-i. It is clear from these figures that the amplitude of the $\mathrm{Nu}$ signal and the average Nusselt number increases with increasing Re, and increases much more with Pr.

The surface averaged value of the Nusselt number is evaluated as

$\mathrm{Nu}=\bar{h} D / k=\frac{1}{S} \int_{S} \mathrm{Nu}_{L} \cdot d S$.

As with the drag coefficient, the time-mean value of the Nusselt number is obtained by considering at least 10 cycles of vortex shedding in periodic unsteady flow regime. The variation in the value of the average Nusselt number with Re in both steady and unsteady flow regimes, for $\operatorname{Re}=50-200$ and $\operatorname{Pr}=0.7,10$ and 100, is presented in Fig. 9. For the range of conditions examined, there is an increase in the average Nusselt number with increasing Re, and a more pronounced increase with increasing $\mathrm{Pr}$, as can be predicted from Fig. 8. The variation of the average Nusselt number for long unconfined semi-circular, trapezoidal, triangular and square cylinders shows the same trend [16,27-32].

Further, the augmentation in heat transfer is calculated by utilizing the following expression (5)

$\mathrm{Nu}(\mathrm{Pr}=100$ or $\mathrm{Pr}=10) / \mathrm{Nu}(\mathrm{Pr}=0.7)$

It is calculated that the ratio of $\mathrm{Nu}$ values belonging to $\mathrm{Pr}=10$ and those belonging to $\operatorname{Pr}=0.7$ ranges from 2.75 to 2.8; however, the ratio of $\mathrm{Nu}$ values belonging to $\operatorname{Pr}=100$ and those belonging to $\operatorname{Pr}=0.7$ ranges from ranges from 6.3 to 6.5 over the Reynolds number domain investigated.

Finally, the present heat transfer results are correlated (Eq. (6)) to calculate the average Nusselt number for the intermediate values of $\mathrm{Re}$ and $\mathrm{Pr}$ in both steady and unsteady regimes for $50 \leqslant \operatorname{Re} \leqslant 200$ and $\operatorname{Pr}=0.7,10$ and 100 .

$\mathrm{Nu}=\left(0.7961 \mathrm{Pr}^{0.416}\right)(1+\operatorname{Re})^{\left(0.472 \mathrm{Pr}^{-0.022}\right)}$

The above correlation has a maximum deviation of less than $5.7 \%$ for $\operatorname{Re}=70$ and $\operatorname{Pr}=0.7$; however, the average deviation is less than $2.9 \%$ for $\operatorname{Re}=50-200$ and $\operatorname{Pr}=0.7-100$, with the present computed heat transfer results. Similar heat transfer correlations have also been established in the literature for cylinders with triangular [42] and square [43] cross-sections for a fixed blockage ratio of $25 \%$.

\subsection{Strouhal number}

The Strouhal number St as a dimensionless vortex shedding frequency can be calculated as $S t=f D / U_{\infty}$. The frequency of the vortex shedding $(f)$ at various values of Re is calculated by Fast Fourier Transform (FFT) of the temporal variation of the lift coefficient. The variation in the value of the Strouhal number with Re is shown in Fig. 10. As the value of Re gradually increases (from $R e=70$ to 200), the value of the Strouhal number increases monotonically. This is also in line with results for various sharp-edged bluff bodies of trapezoidal, square and triangular cross-sections in unconfined domains $[27,28,31]$. Unsurprisingly, the Strouhal number is zero in the steady state ( $\mathrm{Re} \leqslant 69)$ due to the symmetrical attachment of vortices to the downstream of the semi-circular cylinder.

Next, based on the literature [42,43], a simple expression for the Strouhal number was developed as a function of $\operatorname{Re}$ (for the $\operatorname{Re}$ range $70-200$ ).

St $=0.1589 /(1-0.0007 \mathrm{Re})$

The above correlation has a maximum deviation of less than $1.6 \%$ for $\operatorname{Re}=70$; however, the average deviation is less than $1.1 \%$ over the range $70<\mathrm{Re} \leqslant 200$ with the computed St results.

\section{Concluding remarks}

Constant property Newtonian fluid flow around and heat transfer from a semi-circular cylinder are investigated in confined (blockage ratio of 25\%) steady and (periodic) unsteady flow regimes $(\operatorname{Re}=50-200$ and $\operatorname{Pr}=0.7,10$ and 100$)$ by carrying out 2D simulations. A number of flow and heat transfer parameters, such as drag and lift coefficients, Strouhal number and average Nusselt number are studied. The onset of vortex shedding occurs between $\operatorname{Re}=69$ and 70 . The drag coefficient decreases sharply as the Re increases up to 140 and thereafter it increases moderately with increasing Re. The Strouhal number increases with increasing $\mathrm{Re}$ for the range $70 \leqslant \mathrm{Re} \leqslant 200$. The average Nusselt number increases somewhat with Re and substantially with $\mathrm{Pr}$ for the entire range of $\mathrm{Re}$ and $\mathrm{Pr}$ studied. The ratio of $\mathrm{Nu}$ values belonging to $\operatorname{Pr}=100$ and those belonging to $\operatorname{Pr}=0.7$ ranges from 6.3 to 6.5; Prandtl number has a large effect on heat transfer. Finally the correlations of drag coefficient, Strouhal number and average Nusselt number are determined, thus enabling their estimation in other applications at the blockage ratio of $25 \%$.

Future research could concentrate on the effect of blockage and extend the investigation to non-Newtonian fluids.

\section{Acknowledgements}

The authors would like to thank two anonymous reviewers for their valuable comments on this work. The third author gratefully acknowledges the support of the TÁMOP-4.2.1.B-10/2/KONV-201 0-0001 project in the framework of the New Hungarian Development Plan. The realization of this project is supported by the European Union, co-financed by the European Social Fund.

\section{References}

[1] D.S. Steinberg, Cooling Techniques for Electronic Equipment, second ed., Wiley, New York, 1991.

[2] R.P. Chhabra, Hydrodynamics of non-spherical particles in non-Newtonian fluids, in: N.P. Cheremisinoff, P.N. Cheremisinoff (Eds.), Handbook of Applied Polymer Processing Technology, Marcel Dekker, New York, 1996 (Chapter 1).

[3] M.M. Zdravkovich, Flow Around Circular Cylinders: Fundamentals, Oxford University Press, New York, 1997, p. 1.

[4] J.E. Hesselgreaves, Compact Heat Exchangers, Pergamon Press, Oxford, 2001.

[5] R.P. Chhabra, Fluid flow and heat transfer from circular and non-circular cylinders submerged in non-Newtonian liquids, Adv. Heat Transfer 43 (2011) 289-417.

[6] A. Kumar, A. Dhiman, Laminar flow and heat transfer phenomena across a confined semi-circular bluff body at low Reynolds numbers, Heat Transfer Eng. 36 (2015) 1540-1551.

[7] A. Kumar, A. Dhiman, L. Baranyi, CFD analysis of power-law fluid flow and heat transfer around a confined semi-circular cylinder, Int. J. Heat Mass Transfer 82 (2015) 159-169.

[8] M.K. Sukesan, A.K. Dhiman, Laminar mixed convection in a channel with a built-in semi-circular cylinder under the effect of cross-buoyancy, Int. Commun. Heat Mass Transfer 58 (2014) 25-32.

[9] M. Kiya, M. Arie, Viscous shear flow past small bluff bodies attached to a plane wall, J. Fluid Mech. 69 (1975) 803-823.

[10] N. Boisaubert, M. Coutanceau, P. Ehrmann, Comparative early development of wake vortices behind a short semicircular-section cylinder in two opposite arrangements, J. Fluid Mech. 327 (1996) 73-99.

[11] M. Coutanceau, C. Migeon, P. Ehrmann, Particulars of the cross and spanwise near-wake development of a short semicircular-section shell, through the transition Re-range $(60<\mathrm{Re}<600)$, J. Vis. 3 (2000) 9-26.

[12] T. Sophy, H. Sada, D. Bouard, Calcul de I'ecoulementautour d'un cylinder semicirculaire par une method de collocation, CR Mec. 330 (2002) 193-198.

[13] M. Koide, S. Tomida, T. Takahashi, L. Baranyi, M. Shirakashi, Influence of crosssectional configuration on the synchronization of Karman vortex shedding with the cylinder oscillation, JSME Int J., Ser. B 45 (2002) 249-258.

[14] M. Koide, T. Takahashi, M. Shirakashi, Influence of cross-sectional configuration on Karman vortex excitation, J. Comp. Appl. Mech. 5 (2004) 297-310.

[15] A. Chandra, R.P. Chhabra, Influence of power-law index on transitional Reynolds numbers for flow over a semi-circular cylinder, Appl. Math. Model. 35 (2011) 5766-5785.
419

420

421

422

423

424

425

426

427

428
429

430

431

432

433

434

435

436

437

438

439
440

441

442

443

444

445 
HIT 13390

No. of Pages 9, Model 5G

22 June 2016

A. Kumar et al./International Journal of Heat and Mass Transfer $x x x$ (2016) $x x x-x x x$

9

458 [16] A. Chandra, R.P. Chhabra, Momentum and heat transfer from a semi-circular cylinder to power-law fluids in the vortex shedding regime, Numen. Heat Transfer A 63 (2013) 489-510.

[17] A. Gode, A.K. Sahu, R.P. Chhabra, Two-dimensional steady flow over a semicircular cylinder: drag coefficient and Nusselt number, Int. J. Adv. Eng. Sci. Apply. Math. 3 (2011) 44-59.

[18] A.P.S. Binder, S. Sarkar, A. DalaI, Flow over and forced convection heat transfer around a semi-circular cylinder at incidence, Int. J. Heat Mass Transfer 55 (2012) 5171-5184.

[19] D. Chatterjee, B. Mondal, P. Holder, Unsteady forced convection heat transfer over a semi-circular cylinder at low Reynolds numbers, Numen. Heat Transfer A 63 (2013) 411-429.

[20] L.K. Forbes, L.W. Schwartz, Free-surface flow over a semi-circular obstruction, J. Fluid Mech. 114 (1982) 299-314.

[21] A. Chandra, R.P. Chhabra, Flow over and forced convection heat transfer in Newtonian fluids from a semi-circular cylinder, Int. J. Heat Mass Transfer 54 (2011) 225-241.

[22] A. Chandra, R.P. Chhabra, Momentum and heat transfer characteristics of a semi-circular cylinder immersed in power-law fluids in the steady flow regime, Int. J. Heat Mass Transfer 54 (2011) 2734-2750.

[23] A.K. Tiwari, R.P. Chhabra, Momentum and heat transfer characteristics for the flow of power-law fluids over the semi-circular cylinder, Numen. Heat Transfer A 66 (2014) 1365-1388.

[24] D. Chatterjee, B. Mondale, Effect of thermal buoyancy on fluid flow and heat transfer across a semicircular cylinder in cross-flow at low Reynolds numbers, Numen. Heat Transfer A 67 (2015) 436-453.

[25] C.P. Kothandaraman, S. Subramanyan, Heat and Mass Transfer Data Book, eighth ed., New Age International Publishers, 2014.

[26] ANSYS, User Manual, Ansys, Inc., Canonsburg, PA, 2009.

[27] A. Dhiman, M. Masan, Flow and heat transfer over a trapezoidal cylinder: steady and unsteady regimes, Asia-Pac. J. Chem. Eng. 8 (2013) 433-446.

[28] A.K. Sahu, R.P. Chhabra, V. Eswaran, Forced convection heat transfer from a heated square cylinder to power-law fluids in the unsteady flow regime, Numer. Heat Transfer A 56 (2009) 109-131.

[29] V.K. Patnana, R.P. Bharti, R.P. Chhabra, Two-dimensional unsteady flow of power-law fluids over a cylinder, Chem. Eng. Sci. 64 (2009) 2978-2999.
[30] V.K. Patnana, R.P. Bharti, R.P. Chhabra, Two-dimensional unsteady forced convection heat transfer in power-law fluids from a cylinder, Int. J. Heat Mass Transfer 53 (2010) 4152-4167.

[31] D. Chatterjee, Triggering vortex shedding by superimposed thermal buoyancy around bluff obstacles in cross-flow at low Reynolds numbers, Numen. Heat Transfer A 61 (2012) 800-806.

[32] S. Bijjam, A.K. Dhiman, CFD analysis of two-dimensional non-Newtonian power-law flow across a circular cylinder confined in a channel, Chem. Eng. Common. 199 (2012) 767-785.

[33] S. Srikanth, A.K. Dhiman, S. Bijjam, Confined flow and heat transfer across a triangular cylinder in a channel, Int. J. Therm. Sci. 49 (2010) 2191-2200.

[34] T. Rasool, A. Dhiman, M. Parveez, Cross-buoyancy mixed convection around a confined triangular bluff body, Numen. Heat Transfer A 67 (2015) 454-475

[35] H. Abbassi, S. Turki, S.B. Nasrallah, Numerical investigation of forced convection in a plane channel with a builtin triangular prism, Int. J. Therm. Sci. 40 (2001) 649-658.

[36] M. Farhadi, K. Sedighi, A.M. Korayem, Effect of wall proximity on forced convection in a plane channel with a builtin triangular cylinder, Int. J. Therm. Sci. 49 (2010) 1010-1018.

[37] A. Mohsenzedh, M. Farhadi, K. Sedighi, Convective cooling of tandem heated triangular cylinders placed in a channel, Therm. Sci. 14 (2010) 183-197.

[38] G. Biswas, H. Laschefski, N.K. Mitra, M. Fiebig, Numerical investigation of mixed convection heat transfer in a horizontal channel with a builtin square cylinder, Numer. Heat Transfer A 18 (1990) 173-188.

[39] D. Barkley, R.D. Henderson, Three-dimensional Floquet stability analysis of the wake of a circular cylinder, J. Fluid Mech. 322 (1996) 215-241.

[40] A. Chandra, R.P. Chhabra, Mixed convection from a heated semi-circular cylinder to power-law fluids in the steady flow regime, Int. J. Heat Mass Transfer 55 (2012) 214-234.

[41] A. Kumar, A.K. Dhiman, R.P. Bharti, Power-law flow and heat transfer over an inclined square bluff body: effect of blockage ratio, Heat Transfer-Asian Res. 43 (2013) 167-196.

[42] O. Zeitoun, M. Ali, A. Nuhait, Convective heat transfer around a triangular cylinder in an air cross flow, Int. J. Therm. Sci. 50 (2011) 1685-1697.

[43] S. Turki, H. Abbassi, S.B. Nasrallah, Two-dimensional laminar fluid flow and heat transfer in a channel with a builtin heated square cylinder, Int. J. Therm. Sci. 42 (2003) 1105-1113.
494

495

496

497
498

499

500

501

502

504

505
506

507
508

509

510

511

512
513

514
515

515
516

517
518

519

520
521

522

524

525
526

527
528

529

530

531

Please cite this article in press as: A. Kumar et al., Fluid flow and heat transfer around a confined semi-circular cylinder: Onset of vortex shedding

and effects of Reynolds and Prandtl numbers, Int. J. Heat Mass Transfer (2016), http://dx.doi.org/10.1016/j.ijheatmasstransfer.2016.06.026 